\title{
Pediatric Crohn Disease: Clinical and Genetic Characteristics in Taiwan
}

\author{
*Cheng-Hui Hsiao, †Shu-Chen Wei, †Jau-Min Wong, †Hong-Shiee Lai, \\ *Mei-Hwei Chang, and *Yen-Hsuan Ni \\ Departments of ${ }^{*}$ Pediatrics, $\dagger$ Internal Medicine, and $\hbar$ Pediatric Surgery, \\ National Taiwan University Hospital, Taipei, Taiwan
}

\begin{abstract}
Objectives: Crohn disease (CD) is a heterogeneous disorder. The nucleotide oligomerization domain 2/caspase activating recruitment domain 15 (NOD2/CARD15) gene located at 16q12 is strongly associated with susceptibility to $\mathrm{CD}$ in white people but is absent in adult Asian patients, whereas the role of Tolllike receptor 4 (TLR4) polymorphisms has also been reported. Because clinical and genetic data in Asian children with CD are lacking, the aim of this study was to elucidate the clinical and genetic characteristics of Taiwanese children with CD.

Patients and Methods: All of the children hospitalized at the National Taiwan University Hospital between January 2000 and July 2005 who fulfilled the diagnostic criteria for CD were enrolled. Their clinical characteristics were recorded, and genomic DNA was extracted from their white blood cells. After polymerase chain reaction was performed, direct sequencing was done to detect the 4 NOD2 hotspot mutations (P268S, R702W, G908R, 1007fs) and TLR4 polymorphisms (Asp299Gly, Thr399Ile).
\end{abstract}

Results: CD was diagnosed in 10 children ( 6 boys and 4 girls; age range at diagnosis, 14 months to 13 years; median age, 11.1 years). There were 5 children with ileocolonic region involvement, 3 with colonic region involvement, 2 with ileal region involvement, 4 with additional upper gastrointestinal tract involvement, and 2 with additional perianal fistula. Half of the children had growth retardation at diagnosis. Neither NOD2/ $C A R D 15$ mutations nor TLR4 polymorphisms were found in the 10 patients.

Conclusions: Ileocolonic location and inflammatory behavior constitute the most frequent phenotype of $\mathrm{CD}$ in Taiwan. Mutations in the NOD2/CARD15 and TLR4 genes that are common in the West are not associated with CD in Taiwanese children.JPGN 44:342-346, 2007. Key Words: Crohn diseaseNOD2/CARD15-Toll-like receptor 4-Children. (C) 2007 by European Society for Pediatric Gastroenterology, Hepatology, and Nutrition and North American Society for Pediatric Gastroenterology, Hepatology, and Nutrition

\section{INTRODUCTION}

Crohn disease (CD) is a chronic intestinal inflammatory disorder whose pathogenesis and etiology are still largely unknown. Accumulating evidence suggests that it is a heterogeneous disease composed of host genetic components and environmental factors that trigger abnormal immune responses $(1,2)$. Genome-wide linkage analysis and positional cloning have detected the gene responsible for $\mathrm{CD}$, nucleotide oligomerization domain 2 (NOD2), which was later renamed caspase-activating recruitment domain 15 (CARD15) (3-5).

Received August 25, 2006; accepted October 20, 2006.

Address correspondence and reprint requests to Yen-Hsuan Ni, MD, $\mathrm{PhD}$, Department of Pediatrics, National Taiwan University Hospital, No. 7, Chung-Shan South Road, Taipei, Taiwan (e-mail: yhni@ha.mc. ntu.edu.tw).
Four single-nucleotide polymorphisms in the leucinerich region of the NOD2/CARD15 gene on chromosome $16 \mathrm{q} 12$ have been reported to be the hotspots of NOD2/ CARD15mutations, including 1 frameshift cytosineinsertion mutation in exon 11 at position 3020 (3020insC/1007fs) encoding a truncated protein and 2 missense mutations, a C-to-T substitution in exon 4 at position 802 (C802T/P268S), a C-to-T substitution in exon 4 at position 2104(C2104T/R702W), and a G-to-C substitution in exon 8 at position 2722(G2722C/G908R) resulting in an amino acid substitution (4-6).

The Toll-like receptor 4 (TLR4), the main receptor for lipopolysaccharide of gram-negative bacteria, is expressed in macrophages and in dendritic, endothelial, and intestinal epithelial cells, which are partly tolerant to lipopolysaccharide (7-8). TLR4 prevents an exaggerated immune response caused by the large number of bacteria in the intestinal lumen and is suggested to play an important role in the pathogenesis of inflammatory bowel 
disease. Recently, 2 TLR4 polymorphisms (Asp299Gly and Thr399Ile) have been described, with a 2-fold increase in the frequency of the TLR4 Asp299Gly in CD patients as compared with healthy control individuals (9).

Several genotype-phenotype studies have linked NOD2/CARD15 mutations to $\mathrm{CD}$ affecting the ileum and to an early onset of the disease $(6,10-12)$. Patients with homozygous NOD2 mutations represent $34 \%$ of those with an onset before 10 years of age, compared with $3 \%$ with an onset beyond 40 years. This fact justifies the need to study NOD2 mutations in an early-onset population (13). A series of studies showed that the NOD2/CARD15 mutations were present in $25 \%$ to $40 \%$ of European patients with CD $(6,10,12,14-15)$.

However, subsequent studies revealed a lack of NOD2/ CARD15 mutations in Japanese (16), Korean (17), and Chinese (18) patients with CD. It had also been reported that significantly lower frequencies of NOD2/CARD15 mutations were seen in African American and Hispanic children with CD than in white children with CD (19). Although CD is relatively uncommon in Asia, there is a rising trend in Japan and Taiwan $(16,20)$. The aim of this study was to clarify the clinical and genetic characteristics of children with CD in Taiwan.

\section{PATIENTS AND METHODS}

Between January 2000 and July 2005, 10 children with diagnoses of $\mathrm{CD}$ in the National Taiwan University Hospital, a tertiary medical referral center with an annual admission of approximately 7000 pediatric patients, were recruited. Their clinical data were collected, including age at diagnosis, interval from onset of disease to diagnosis, symptoms, and abnormal laboratory data at CD diagnosis. History of laparotomy and/or appendectomy, and the presence of fistula, amenorrhea, and growth retardation defined as body weight and/or body height less than the third percentile of normal population in Taiwan, were also checked.

The anatomic distribution of the disease at the time of diagnosis and during follow-up, and the disease behavior, were determined by endoscopic and/or radiologic evaluation and/or surgical intervention. Follow-up began at the time of diagnosis and ended in September 2005.

DNA for sequence analysis was extracted from the patients' white blood cells. The study protocol was approved by the institutional review board of the hospital's ethics committee.

\section{Criteria for CD Diagnosis}

All of the patients underwent colonoscopic examination with multiple biopsies, and 9 underwent upper endoscopy or upper gastrointestinal (GI) tract series of barium radiographs. For evaluation of the terminal ileum, small bowel followthrough barium radiographs and/or colonoscopy and ileoscopy were performed. Stool studies were also checked in all cases to exclude parasitic and bacterial causes. A diagnosis of $C D$ required the presence of at least 2 of the following: history of diarrhea and/or abdominal pain for more than 3 months, endoscopic identification of segmental lesions of cobblestone appearance and/or radiologic evidence of stenosis in the small and/or large bowel, histological findings of transmural lymphocytic infiltration and/or occurrence of epithelial granulomas with giant cells of the Langhans type (21), and the presence of fistulas and/or abscesses in relation to the intestinal lesion (22).

\section{Anatomic Extent of the Disease and Disease Behavior}

All of our cases were categorized in 1 of the following 3 groups, depending on the anatomic extent of the disease: ileal, with gross involvement of the ileum without colonic disease; ileocolonic, with gross involvement of both the ileum and the colon; and colonic, with gross involvement of the colon without small bowel involvement. In addition, 2 separate categories could be added to any of the aforementioned groups: perianal disease, with perianal fistulas and/or perianal abscesses, not merely perianal fissures or tags; and upper GI tract involvement, with gross involvement of the upper GI tract, including the esophageal, gastric, or duodenal regions.

In each case, all areas of anatomic involvement were confirmed by histological evidence, except for jejunal and perianal lesions. Moreover, all of our patients were categorized into 1 of the 3 groups by the Vienna classification guidelines criteria (23): fistulizing (penetrating), stricturing (fibrostenosing), and inflammatory disease behavior. We also evaluated the patients' conditions at the time of diagnosis and at the end of follow-up according to the Pediatric Crohn Disease Activity Index (PCDAI) scores (24). The minimum total index score is 0 , and the maximum is 100 . Inactive, mild, moderate, and severe disease were defined as PCDAI scores $\leq 15,16-30,31-45$, and $>45$, respectively.

\section{DNA Purification From Whole Blood and Genotyping}

Genomic DNA was purified from whole blood with use of the Genomic DNA Mini Kit (Geneaid, Tao-Yuan, Taiwan) according to the manufacturer's instruction. With a GeneAmp PCR System 9600 thermal cycler (Applied Biosystems, Foster City, CA), polymerase chain reaction (PCR) with appropriate primers (Table 1) was performed with an initial denaturation at $94^{\circ} \mathrm{C}$ for 5 minutes, followed by 35 cycles of denaturing at $94^{\circ} \mathrm{C}$ for 1 minute, annealing at $50^{\circ} \mathrm{C}$ in $N O D 2$ analysis and at $58^{\circ} \mathrm{C}$ in TLR4 analysis for 2 minutes, extension at $72^{\circ} \mathrm{C}$ for 1 minute, and final incubation at $72^{\circ} \mathrm{C}$ for 7 minutes. The PCR products were purified by use of a PCR purification kit (Geneaid, Tao-Yuan, Taiwan) according to the manufacturer's instructions.

After PCR, direct sequencing was performed at the specific regions of the NOD2 gene, including a frame-shift $\mathrm{C}$-insertion mutation 3020ins $\mathrm{C} / 1007 \mathrm{fs}$ in exon 11 ; and 3 missense mutations, 802C-to-T/P268S in exon 4, 2104C-to-T/R702W in exon 4 , and $2722 \mathrm{G}-$ to-C/G908R in exon 8 . In addition, 2 nucleotide polymorphisms of the TLR4 gene resulting in the Asp299Gly and Thr399Ile amino acid substitutions were determined by use of a previously described assay (25) that creates specific NcoI and HinfI restriction sites for the TLR4 Asp299Gly and Thr399Ile polymorphisms, respectively. If no restricted bands occurred, additional PCR followed by direct sequencing was also performed to confirm the results. The sequence primers were the same as those in the PCR 
TABLE 1. Primers used for the polymerase chain reaction (PCR) of NOD2 and TLR4 genes

\begin{tabular}{llll}
\hline Primers & & Sequence & PCR product size \\
\hline NOD2 & SNP5(F) & $5^{\prime}$-tgc,ctc,ttc,ttc,tgc,ctt,cc-3' & 422 bp \\
& SNP5(R) & $5^{\prime}$-agt,aga,gtc,cgc,aca,gag,ag-3' & 437 bp \\
& R702W(F) & $5^{\prime}$-gtg,ctt,ctt,tgc,cgc,gtt,cta,c-3' & 345 bp \\
& R702W(R) & $5^{\prime}$-gct,cct,cct,gca,tct,cgt,aca,g-3' & \\
& G908R(F) & $5^{\prime}$-gtg,agg,cca,ctc,tgg,gat,tga,g-3' & 533 bp \\
& G908R(R) & $5^{\prime}$-ttt,cct,tac,tcc,att,gcc,taa,c-3' & \\
TLR4 sequencing & 1007fs(F) & $5^{\prime}$-ctg,agc,ctt,tgt,tga,tga,gc-3' & 619 bp \\
& $1007 f(\mathrm{f})$ & $5^{\prime}$-tct,tca,acc,aca,tcc,cca,tt-3' & \\
\hline
\end{tabular}

amplifications. Last, the cycle-sequencing products were purified by ethanol precipitation and analyzed by Genetic Analyzer ABI PRISM 3100 (Applied Biosystems, Alexandria, VA) according to the manufacturer's instructions.

\section{Statistical Analysis}

The Fisher exact test for categorical variables and the Student $t$ test for continuous variables were used. $P<0.05$ was considered statistically significant.

\section{RESULTS}

\section{Clinical Manifestations}

From January 2000 to July 2005, 10 children with diagnoses of $C D$ were enrolled in our study (the male: female ratio was 6:4). The median age at diagnosis was 11.1 years (range 1.2-13.2 years), and half had onset before the age of 10 years. The median interval from onset of disease to diagnosis was 9.0 months (range 3-36 months). The clinical manifestations and phenotype of each patient are given in Table 2.

The clinical symptoms, signs, and laboratory results in the 10 children at diagnosis are summarized in Table 3.
Four patients had extraintestinal symptoms, such as skin rash, arthralgia, and episcleritis. The family history was positive in 1 patient $(10 \%)$, whose maternal grandmother had also received a diagnosis of $\mathrm{CD}$.

Three (30\%) patients had CD associated with other diseases, including gallstones in 1 , unilateral congenital hydroureter in 1, and incontinentia pigmenti and urolithiasis of calcium oxalate in 1 . Four (40\%) patients had been breast-fed for at least 1 week after birth. None had a significant delay of developmental milestones at diagnosis, except 1 with language delay. Before the diagnosis of $\mathrm{CD}$, antituberculous therapy had been received by $2(20 \%)$ patients, appendectomy by 2 , laparotomy by 3 , and antiamoeba therapy by 1 .

\section{Anatomic Extent of the Disease and Disease Behavior}

According to the anatomic involvement of $\mathrm{CD}$, the most common disease location was the ileocolonic region in 5 patients (50\%), colonic region in 3, ileal region in 2 , perianal disease in 2, and upper GI involvement in 4. In the aspect of disease behavior according to the Vienna classification (23), fistulizing disease was identified in

TABLE 2. Summary of the pediatric Crohn disease patients in this study

\begin{tabular}{|c|c|c|c|c|c|c|c|c|c|}
\hline Patient & Sex & Age, y & $\begin{array}{l}\text { Age at } \\
\text { onset, } y\end{array}$ & $\begin{array}{c}\text { Age at } \\
\text { diagnosis, y }\end{array}$ & $\begin{array}{c}\text { Duration } \\
\text { between onset } \\
\text { and diagnosis, mo }\end{array}$ & Site and extent of disease & $\begin{array}{l}\text { Disease } \\
\text { behavior }\end{array}$ & $\begin{array}{c}\text { PCDAI } \\
\text { scores at } \\
\text { diagnosis }\end{array}$ & $\begin{array}{l}\text { PCDAI scores } \\
\text { at end of } \\
\text { follow-up }\end{array}$ \\
\hline 1 & M & 12.9 & 12.3 & 12.9 & 7 & Ileum & Inflammatory & 47.5 & 5 \\
\hline 2 & $\mathrm{~F}$ & 11.3 & 9.5 & 11.2 & 20 & Ileum + upper GI & Inflammatory & 40 & 7.5 \\
\hline 3 & M & 12.1 & 10.8 & 12.1 & 16 & Colon & Inflammatory & 45 & 0 \\
\hline 4 & $\mathrm{~F}$ & 12.3 & 11.7 & 12.1 & 5 & Colon + upper GI & Inflammatory & 60 & 5 \\
\hline 5 & $\mathrm{~F}$ & 14.3 & 12.8 & 13.2 & 4 & Ileum + colon & Inflammatory & 52.5 & 0 \\
\hline 6 & M & 5.1 & 0.3 & 1.2 & 11 & Ileum + colon + upper GI & Inflammatory & 50 & 5 \\
\hline 7 & M & 25.2 & 4.1 & 4.5 & 5 & Ileum + colon + perianal & Fistulizing $^{\dagger}$ & 65 & 17.5 \\
\hline 8 & M & 16.3 & 10.8 & 11.0 & 3 & Ileum + colon & Fistulizing & 70 & 10 \\
\hline 9 & $\mathrm{~F}$ & 24.8 & 7.8 & 10.8 & 36 & Ileum + colon & Fistulizing $^{\dagger}$ & 55 & 25 \\
\hline 10 & M & 4.8 & 2.5 & 3.6 & 13 & Colon + upper GI + perianal & Fistulizing $^{\dagger}$ & 65 & 10 \\
\hline
\end{tabular}

PCDAI indicates Pediatric Crohn Disease Activity Index; GI, gastrointestinal.

* According to Vienna classification guidelines criteria.

${ }^{\dagger}$ The patient had received bowel resection. 
TABLE 3. Clinical symptoms, signs, and laboratory findings in 10 children with $C D$ at diagnosis

\begin{tabular}{lr}
\hline Clinical manifestations & Patient \\
\hline Symptoms and signs & \\
Abdominal pain & 10 \\
Diarrhea & 6 \\
Rectal bleeding & 6 \\
Vomiting & 3 \\
Oral ulcer & 3 \\
Weight loss & 6 \\
Fever & 5 \\
Extraintestinal symptoms/signs & 4 \\
Skin rash & 3 \\
Arthralgia/arthritis & 2 \\
Episcleritis & 1 \\
Fistula & 4 \\
Growth retardation & 5 \\
Body weight $<3 \mathrm{rd}$ percentile & 4 \\
Body height $<3 \mathrm{rd}$ percentile & 3 \\
Amenorrhea & 1 \\
History of laparotomy/appendectomy & 4 \\
Laboratory findings & \\
ESR $>20 \mathrm{~mm} / \mathrm{h}^{*}$ & 10 \\
Anemia $(\mathrm{Hct})^{* \dagger}$ & 7 \\
CRP $>1.2 \mathrm{mg} / \mathrm{dL}{ }^{*}$ & 8 \\
Albumin $<3.5 \mathrm{~g} / \mathrm{dL}$ & 5 \\
Leukocytosis $>15,000 / \mu \mathrm{L}$ & 6 \\
\hline
\end{tabular}

Hct indicates hematocrit; ESR, erythrocyte sedimentation rate; CRP, C-reactive protein.

${ }^{*}$ The limits are the laboratory reference in the Pediatric Crohn Disease Activity Index.

${ }^{\dagger}$ Male and female $<10$ y, Hct $<33 \%$; 11-14 y male, Hct $<35 \%$; $15-$ 18 y male, Hct $<37 \%$; $11-18$ y female, Hct $<34 \%$.

4 patients, inflammatory disease in 6 , and stricturing disease in none (Table 2). Three patients had undergone surgical resection of the bowel, including either colectomy or ileal resection. Patients with fistulizing behavior had significantly higher potential for the development of growth retardation $(100 \%$ vs $16.7 \%, P=0.024)$ and more need for bowel resection (75\% vs $0 \%, P=0.033)$ than those with inflammatory behavior.

Children with fistulizing CD had a more frequent onset before 10 years of age than those with inflammatory CD ( $75 \%$ vs $33.3 \%, P=0.238$ ). The mean PCDAI scores at diagnosis among patients with fistulizing behavior were $63.8 \pm 6.2$ (range 55-70), which were significantly higher than those among patients with inflammatory behavior $(49.2 \pm 5.5$, range: $40-60, P=0.009)$. Similarly, at the end of follow-up, the mean PCDAI scores among patients with fistulizing behavior were $15.6 \pm 7.0$ (range 10-25), which were also significantly higher than those among patients with inflammatory behavior $(3.8 \pm 2.4$, range $0-7.5, P=0.023)$.

\section{Treatment and Follow-up}

Until September 2005, the mean duration of follow-up was $5.5 \pm 4.7$ years (range $0.3-25.2$ years). Under medical treatment with 5-aminosalicylic acid, corticosteroids, and semielemental diet, occasionally combined with antibiotics (metronidazole) and total or partial parenteral nutrition, all 10 patients survived. Four (40\%) received immunosuppressive drugs (tacrolimus) or immunomodulatory medicine (azathioprine and 6-mercaptopurine) to help control disease activity and taper down the corticosteroids. Three (30\%) patients required surgical intervention, including repeated fistulectomy and resection of either the colon or the ileum.

According to the PCDAI scores, although severe disease was identified at diagnosis in 8 patients $(80 \%)$ and moderate disease in 2 (20\%), most had improved after therapy. Eight $(80 \%)$ had inactive disease and only $2(20 \%)$ had mild disease at the end of follow-up. In addition, although $5(50 \%)$ had growth retardation at diagnosis, most had caught up after treatment, and only 1 experienced persistent growth failure and osteoporosis at the end of follow-up. This patient received multiple fistulectomy and ileoectomy, and she had primary delayed puberty with amenorrhea until she was 24 years old.

\section{Genotyping}

In our study, none of the common mutations of NOD2/ CARD15 gene in whites (P268S, R702W, G908R, 1007fs) and TLR4(Asp299Gly, Thr399Ile) polymorphisms were detected in our Taiwanese children with CD. All of their DNA sequences showed the wild-type in these regions.

\section{DISCUSSION}

Crohn disease is a heterogeneous disease with different causes of genetic background and environmental factors. There are various clinical presentations among different ethnic groups. In our study the characteristics of Taiwanese children with $\mathrm{CD}$ included ileocolonic involvement as the most common phenotype (50\%), male predominance (M:F 1.5:1), and rare familial aggregation.

Polito et al (26) showed that if the diagnosis was made before the age of 20, CD usually ran a more complicated course, including a higher incidence of small bowel involvement, more bowel strictures, and more chances of surgical intervention. In our study half of the children with CD had early onset at less than 10 years of age and $70 \%$ had small bowel involvement. However, only $30 \%$ needed surgery for bowel resection, whereas $40 \%$ needed other immunosuppressive or immunomodulatory medicine to complement the corticosteroid treatment. Furthermore, the severity of CD in our children according to PCDAI scores seemed higher than in the Western reports (22). Growth retardation was common in children with inflammatory bowel disease, particularly CD (27). In our study, although $50 \%$ had growth retardation at diagnosis, most caught up later after adequate treatment. 
The recent discovery of NOD2/CARD15 on chromosome 16 explains approximately $20 \%$ of the genetic susceptibility in Western countries (13). Several studies have shown that NOD2/CARD15 mutations were strongly associated with $\mathrm{CD}$, in particular affecting the ileum and having earlier onset $(6,10-12)$. Most of our patients had the characteristics of early onset, great proportion of ileal involvement, and severe disease activity at diagnosis. These manifestations led us to speculate that there would be a high proportion of CARD15 mutations. However, CARD15 mutations were not the cause. Thus, genetic susceptibility of CD seems to vary in different ethnic populations.

Brand et al (9) showed that approximately $15 \%$ of 204 German patients with CD were heterozygous for the TLR4 mutations (Asp299Gly and Thr399Ile), suggesting that $T L R 4$ polymorphisms play a role in a minority of patients with CD. In our study, none had TLR4 mutations. Some specific regions of class II HLA on chromosome 6, which were associated with susceptibility to $\mathrm{CD}$, had been identified among the Japanese population and in whites $(28,29)$. Whether this gene plays an important role in Asian children with CD remains unknown. The susceptibility gene of $\mathrm{CD}$ in Asian people mandates additional studies.

A limitation of this study is that we surveyed only the hotspots of NOD2/CARD15 genes instead of the fulllength gene sequencing. Hence, the possibility of variants other than the 4 hotspots in this gene cannot be excluded.

In conclusion, ileocolonic involvement and inflammatory behavior were the characteristics of Taiwanese children with $\mathrm{CD}$, but mutations of NOD2/CARD15 and TLR4 genes were not found.

\section{REFERENCES}

1. Sartor RB. Microbial Factors in the Pathogenesis of Crohn's Disease, Ulcerative Colitis, and Experimental Intestinal Inflammation. Philadelphia: WB Saunders; 2000.

2. Dianda L, Hanby AM, Wright LA, et al. T cell receptor-alpha betadeficient mice fail to develop colitis in the absence of a microbial environment. Am J Pathol 1997;150:91-7.

3. Hugot JP, Laurent-Puig P, Gower-Rousseau C, et al. Mapping of a susceptibility locus for Crohn's disease on chromosome 16. Nature 1996;379:821-3.

4. Hugot JP, Chamaillard M, Zouali H, et al. Association of NOD2 leucine-rich repeat variants with susceptibility to Crohn's disease. Nature 2001;411:599-603.

5. Ogura Y, Bonen DK, Inohara N, et al. A frameshift mutation in NOD2 associated with susceptibility to Crohn's disease. Nature 2001;411:603-6.

6. Cuthbert AP, Fisher SA, Mirza MM, et al. The contribution of NOD2 gene mutations to the risk and site of disease in inflammatory bowel disease. Gastroenterology 2002;122:867-74.

7. Cario E, Rosenberg IM, Brandwein SL, et al. Lipopolysaccharide activates distinct signaling pathways in intestinal epithelial cell lines expressing Toll-like receptors. J Immunol 2000;164:966-72.

8. Nomura F, Akashi S, Sakao Y, et al. Cutting edge: endotoxin tolerance in mouse peritoneal macrophages correlates with downregulation of surface toll-like receptor 4 expression. $J$ Immunol 2000;164:3476-9.
9. Brand S, Staudinger T, Schnitzler F, et al. The role of Toll-like receptor 4 Asp299Gly and Thr399Ile polymorphisms and CARD15/NOD2 mutations in the susceptibility and phenotype of Crohn's disease. Inflamm Bowel Dis 2005;11:645-52.

10. Lesage S, Zouali H, Cezard JP, et al. CARD15/NOD2 mutational analysis and genotype-phenotype correlation in 612 patients with inflammatory bowel disease. Am J Hum Genet 2002;70:845-57.

11. Bairead E, Harmon DL, Curtis AM, et al. Association of NOD2 with Crohn's disease in a homogenous Irish population. Eur J Hum Genet 2003;11:237-44.

12. Ahmad T, Armuzzi A, Bunce M, et al. The molecular classification of the clinical manifestations of Crohn's disease. Gastroenterology 2002;122:854-66.

13. Hugot JP, Zouali H, Lesage S. Lessons to be learned from the NOD2 gene in Crohn's disease. Eur J Gastroenterol Hepatol 2003;15: 593-7.

14. Hampe J, Grebe J, Nikolaus S, et al. Association of NOD2 (CARD15) genotype with clinical course of Crohn's disease: a cohort study. Lancet 2002;359:1661-5.

15. Vermeire S, Wild G, Kocher K, et al. CARD15 genetic variation in a Quebec population: prevalence, genotype-phenotype relationship, and haplotype structure. Am J Hum Genet 2002;71:74-83.

16. Inoue N, Tamura K, Kinouchi Y, et al. Lack of common NOD2 variants in Japanese patients with Crohn's disease. Gastroenterology 2002;123:86-91.

17. Croucher PJ, Mascheretti S, Hampe J, et al. Haplotype structure and association to Crohn's disease of CARD15 mutations in two ethnically divergent populations. Eur J Hum Genet 2003;11: $6-16$.

18. Guo QS, Xia B, Jiang Y, et al. NOD2 3020insC frameshift mutation is not associated with inflammatory bowel disease in Chinese patients of Han nationality. World J Gastroenterol 2004;10: $1069-71$.

19. Kugathasan S, Loizides A, Babusukumar U, et al. Comparative phenotypic and CARD15 mutational analysis among African American, Hispanic, and white children with Crohn's disease. Inflamm Bowel Dis 2005;11:631-8.

20. Tsai $\mathrm{CH}$, Chen $\mathrm{HL}, \mathrm{Ni} \mathrm{YH}$, et al. Characteristics and trends in incidence of inflammatory bowel disease in Taiwanese children. J Formos Med Assoc 2004;103:685-91.

21. Morson BC. Histopathology of Crohn's disease. Proc R Soc Med 1968;61:79-81.

22. Langholz E, Munkholm P, Krasilnikoff PA, et al. Inflammatory bowel disease with onset in childhood: clinical features, morbidity, and mortality in a regional cohort. Scand $J$ Gastroenterol 1997;32:139-47.

23. Gasche C, Scholmerich J, Brynskov J, et al. A simple classification of Crohn's disease: report of the Working Party for the World Congresses of Gastroenterology, Vienna 1998. Inflamm Bowel Dis 2000;6:8-15.

24. Hyams JS, Ferry GD, Mandel FS, et al. Development and validation of a pediatric Crohn's disease activity index. J Pediatr Gastroenterol Nutr 1991;12:439-47.

25. Lorenz E, Frees KL, Schwartz DA. Determination of the TLR4 genotype using allele-specific PCR. Biotechniques 2001;31:22-4.

26. Polito JM, Childs B, Mellits ED, et al. Crohn's disease: influence of age at diagnosis on site and clinical type of disease. Gastroenterology 1996;111:580-6.

27. Ferguson A, Sedgwick DM. Juvenile onset inflammatory bowel disease: height and body mass index in adult life. $\mathrm{Br}$ Med $J$ 1994;308:1259-63.

28. Brant SR, Panhuysen CIM, Bailey-Wilson JE, et al. Linkage heterogeneity for the IBD1 locus in Crohn's disease pedigrees by disease onset and severity. Gastroenterology 2000;119:1483-90.

29. Nakajima A, Matsuhashi N, Kodama T, et al. HLA-linked susceptibility and resistance genes in Crohn's disease. Gastroenterology 1995;109:1462-7. 